\title{
SENSORES INVASIVOS E NÃO INVASIVOS: CONCEITOS E APLICAÇÕES BIOMÉDICAS
}

\author{
INVASIVE AND NON INVASIVE SENSORS: \\ CONCEPTS AND BIOMEDICAL APPLICATIONS \\ Marcella Wayss Darold ${ }^{1}$, Fernanda Vargas de Almeida ${ }^{2}$, \\ Helena Oliveira Bertolino ${ }^{3}$ e Paulo Jefferson Dias de Oliveira Evald ${ }^{4}$
}

\section{RESUMO}

A Instrumentação Biomédica abrange os estudos sobre o princípio de funcionamento de diversos dispositivos que entram em contato com o corpo humano para diferentes fins na área da saúde. Um dos componentes críticos destes dispositivos são os biossensores, que têm como finalidade mensurar variáveis internas, a partir de métodos invasivos que requerem procedimento cirúrgico e ambiente controlado, em contrapartida aos sensores externos, que mensuram por métodos não invasivos e não possuem interação física ou química direta com o processo. Apesar dos métodos invasivos serem considerados mais precisos, podem trazer complicações consideráveis ao paciente. Como alternativa, os sensores não invasivos têm alcançado cada vez mais espaço no mercado, pois suas aplicações não apresentam tamanho risco, entretanto, possuem como desvantagem o posicionamento manual no paciente para captar as informações, que podem sofrer alterações externas. Assim, neste trabalho é apresentada uma discussão sobre os recentes avanços e aplicações dos sensores biomédicos invasivos e não invasivos, bem como uma discussão sobre as tendências, desafios e perspectivas futuras para esta área.

Palavras-chave: Instrumentação Clínica, Transdutores Biomédicos, Sensores Biomédicos.

\section{ABSTRACT}

Biomedical Instrumentation covers the studies on the working principle of various dispositive that come into contact with the human body for different health purposes. One of the critical components of these devices are the biosensors, which have the purpose of measuring internal variables, from invasive methods that require surgical procedure and controlled environment, in contrast to external sensors, which measure by non-invasive methods and have no direct physical or chemical interaction with the process. Although invasive methods are considered more accurate, they can bring considerable complications to the patient. As an alternative, non-invasive sensors have increasingly reached more space in the market, because their applications do not present such a risk, however, they have the disadvantage of manual positioning in the patient to capture information, which can suffer external changes. Thereby, in this work is presented a discussion about recent developments and applications of biomedical invasive and non-invasive sensors, as well as a discussion on tendencies, challenges and future perspectives to this area.

Keywords: Clinical Instrumentation, Biomedical transducers, Biomedical Sensors.

\footnotetext{
${ }^{1}$ Discente do curso de Engenharia Biomédica da UFN. E-mail: marcelladarold@gmail.com

${ }^{2}$ Discente do curso de Engenharia Biomédica da UFN. E-mail: fernandopolitana@gmail.com

${ }^{3}$ Discente do curso de Engenharia Biomédica da UFN. E-mail: helenabertolino@live.com

${ }^{4}$ Professor Assistente da UFN. E-mail: paulo.evald@ufn.edu.br
} 


\section{INTRODUÇÃO}

A instrumentação biomédica é a área que estuda o funcionamento e projeto dos instrumentos biomédicos em geral. Esses instrumentos são equipamentos elétricos, eletrônicos e/ou mecânicos, que são designados para diagnóstico, tratamento ou monitoração de pacientes, sob supervisão médica, que podem estabelecer contato físico de modo invasivo ou não invasivo (WEBSTER, 2009). Estes dispositivos visam captar informações biológicas do paciente, e realizar diversas operações, tais como: transdução, amplificação, filtragem e armazenamento. Assim, esses dispositivos de mensuração interagem com o tecido, tanto para possibilitar a realização da medida, quanto para aplicar o tratamento. De modo geral, essa variável a ser medida é a quantidade, condição ou propriedade física que é medida pelo sistema de instrumentação (BRONZINO, 2006, DORF, 2018). Essa é uma das áreas mais promissoras da atualidade, pois há uma constante busca por novos sensores, instrumentos e dispositivos biomédicos que possuam mais precisão, segurança, robustez e que forneçam mais conforto aos pacientes.

Em relação aos meios de mensuração, as variáveis medidas podem ser internas, as quais requerem métodos invasivos para sua medição direta, tais como a mensuração dos níveis hormonais e débito cardíaco), ou variáveis de medidas externas, as quais podem ser medidas de forma não invasiva, tais como os potenciais bioelétricos de superfície e ressonância magnética (WANG; LIU, 2011). Logo, os sensores são componentes críticos em qualquer sistema que envolve o monitoramento de grandezas físicas ou químicas (ZHOU; WANG; CUI, 2015), principalmente se o sistema consiste em uma unidade de tratamento biomédico.

Dentro deste contexto, um dos assuntos frequentemente estudados são os biossensores. Os avanços tecnológicos revolucionaram o laboratório clínico ao longo dos anos e, mais recentemente, o desenvolvimento e a miniaturização de sensores eletroquímicos, biossensores e sensores de fibra óptica, permitindo sua viabilidade experimental in vivo (vide (ROOINTAN et al., 2019; CHINNADAYALA et al., 2018; SHI; ANG; ZHAO, 2018; XIAO et al., 2017; LAI et al., 2016)). De modo geral, os biossensores atuam como interface entre um sistema biológico (caracterizado por variáveis fisiológicas) e um sistema eletrônico (caracterizado por sinais elétricos), sendo necessário que suas características de funcionamento sejam adequadas para ambos. Para isso, há dois tipos de sensores: invasivos e não invasivos, onde estes fornecem o monitoramento contínuo da condição do paciente em tempo real (BURRIT, 1998; WEBSTER, 2009).

Os sensores invasivos são aqueles que requerem procedimento cirúrgico e ambiente controlado, pois interagem física e quimicamente com o sistema ao qual são inseridos. Nestes sistemas, destacam-se diversos dispositivos, tais como: marcapassos, desfibriladores implantáveis, monitores para $\mathrm{pH}$ e gases no sangue, que podem usar química de fluorescência. No desenvolvimento do biossensor implantável, uma questão chave adicional é considerar a biocompatibilidade do sensor e a vida útil 
operacional do corpo sabendo que, quando um sensor biomédico é implantado no corpo, ele inevitavelmente entra em contato com os fluidos corporais (ZHOU; WANG; CUI, 2015). A principal tendência dos sensores invasivos são a miniaturização desses dispositivos, tornando-os minimamente invasíveis e mais confortáveis para o paciente, como os seguintes exemplos (XUE et al., 2018; BANERJEE, H.; SHEN, S.; REN, 2018; TAYLOR et al., 2018).

Já os sensores não invasivos não necessitam de cirurgia e não têm interação física ou química com o sistema, dividindo-o em medida direta e indireta. Destaca-se que o método direto normalmente acarreta maior custo e complexidade de implementação. Alguns desses sistemas incluem: eletroencefalogramas, termômetros, oximetrias de pulso, eletrodos transcutâneos para pressão parcial de oxigênio, eletrodos transcutâneos para pressão parcial de gás carbônico e monitores transcutâneos de bilirrubina (BURRIT, 1998; TOGAWA; TAMURA; OBERG, 2011).

Em termos de comparação, brevemente, pode-se realçar algumas vantagens dos sensores invasivos contínuos, tais como: a redução no tempo de resposta terapêutica sem problemas pré-analíticos, sem perda de sangue, diminuição do risco de exposição a patógenos transmitidos pelo sangue e disponibilidade de dados de tendências em tempo real (BURRIT, 1998, RAY, 2018). Entretanto, como desvantagem, existem diversos transdutores invasivos, cuja corrente elétrica a medir é transportada para um enrolamento com certa quantidade de espiras no núcleo, precisando de uma abertura do fio condutor que será medido. Já em modelos não invasivos, os transdutores apresentam um canal para a passagem do fio condutor, que é uma ampla vantagem em relação ao seu correspondente invasivo. Além disso, alguns modelos têm um núcleo dividido que pode se abrir para a instalação do condutor a ser medido, estes dispositivos são denominados transdutores com núcleo bipartido (KOUIZUMI, 2012).

Como pode ser notado nessa discussão inicial, a escolha do tipo de sensor depende da grandeza a ser medida e das vantagens e desvantagens que ele possui, sempre levando em consideração a precisão das medidas, que é fundamental, e o bem estar dos pacientes. Assim, a seguir é apresentada uma discussão sobre recentes trabalhos de ambos os tipos de sensores, invasivos e não-invasivos, em aplicações biomédicas. Em seguida, será apresentada uma discussão sobre as tendências, desafios e perspectivas futuras nessa área.

\section{SENSORES NÃO INVASIVOS E SUAS APLICAÇÕES BIOMÉDICAS}

Os sensores não invasivos não possuem interação física ou química com o processo, podendo ser sensores de temperatura, eletromiografia, eletrocardiograma, glicose, eletromiografia, eletroencefalograma, sensor de pressão sanguínea, entre outros (PRUTCHI; NORRIS, 2005). Um exemplo simples é mostrado na Figura 1, onde são dispostos dois eletrodos sobre a pele do paciente para medir uma determinada diferença de potencial, que poderia ser utilizada por exemplo, no eletrocardiograma. 
Figura 1 - Esquema de medição da diferença de dois biopotenciais de forma não invasiva.

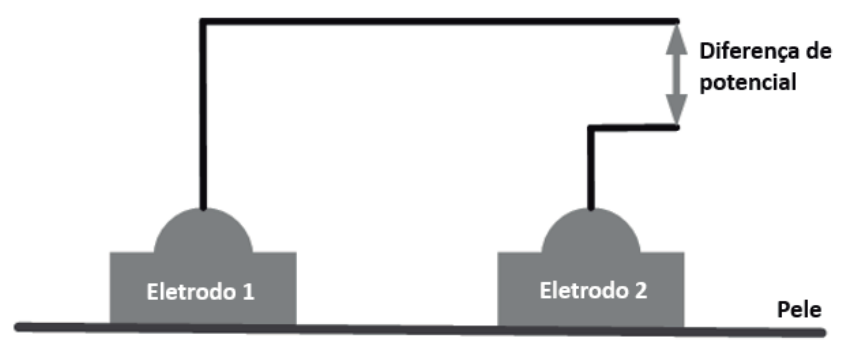

Fonte: Construção dos autores.

Definir se o equipamento será invasivo ou não, é um dos requisitos de projeto do equipamento para, principalmente, planejar o risco de acidentes ou infecções (WEBSTER, 2009). A Tabela 1 mostra de forma resumida os trabalhos que irão ser discutidos nessa secção.

Tabela 1 - Compilação dos trabalhos que abordam avanços em sensores físicos com aplicação biomédica.

\begin{tabular}{|c|c|c|c|}
\hline Tipo de Sensor & Aplicação & Material empregado & Referência \\
\hline Sensor físico & $\begin{array}{l}\text { Medida da bioimpedância do } \\
\text { tórax }\end{array}$ & Eletrodos em forma de fita & $\begin{array}{l}\text { (HENRIQUES, 2017; } \\
\text { SLOMP, 1999) }\end{array}$ \\
\hline Sensor físico & $\begin{array}{l}\text { Medição de pressão arterial } \\
\text { instantânea }\end{array}$ & Bolsa de ar inflada e pletismógrafo fotoelétrico & $\begin{array}{l}\text { (GOMEZ; DIAN; ZEILER, 2020; } \\
\text { JÄÄSKELÄINEN, 2018; } \\
\text { TAPIA et al., 2017; } \\
\text { SLOMP, 1999) }\end{array}$ \\
\hline Sensor físico & Medida pressão sanguínea & $\begin{array}{l}\text { Sensor de pressão pequeno com alta sensibili- } \\
\text { dade e micromanômetro piezo-resistivo }\end{array}$ & $\begin{array}{l}\text { (SLOMP, 1999; } \\
\text { MEIDERT; SAUGEL, 2018) }\end{array}$ \\
\hline Sensor físico & $\begin{array}{l}\text { Medida de oxigênio que o } \\
\text { sangue arterial transporta }\end{array}$ & Fonte de luz, diodos e fotodetector & $\begin{array}{l}\text { (KIM et al, 2017; } \\
\text { VERKRUYSSE et al.,2017; } \\
\text { SANTANA, 2017) }\end{array}$ \\
\hline Sensor vestível & Medida dos sinais físicos & Sensores flexíveis & $\begin{array}{l}\text { (MAJUMDER; MONDAL; } \\
\text { DEEN, 2017; } \\
\text { ZHANG et al., 2020) }\end{array}$ \\
\hline Sensor vestível & $\begin{array}{l}\text { Medida di níveis de lactato, } \\
\text { glicose, álcool e ureia na } \\
\text { transpiração humana }\end{array}$ & $\begin{array}{l}\text { Biossensor eletroquímico flexível baseados em } \\
\text { nanofios de Prata por impressão molecular }\end{array}$ & (ZHANG et al., 2020) \\
\hline Sensor vestível & $\begin{array}{l}\text { Detectar de forma não invasiva } \\
\text { e síncrona a glicose e ureia } \\
\text { excretadas no suor humano }\end{array}$ & $\begin{array}{l}\text { Sensor colorimétrico vestível que é baseado } \\
\text { em fios de algodão, nanofitas de celulose e } \\
\text { óxido de quitosana-grafeno para modificar as } \\
\text { superfícies do fio de algodão }\end{array}$ & (PROMPHET et al., 2020) \\
\hline Sensor vestível & Medidas dos sinais bioelétricos & $\begin{array}{l}\text { Sensores sem fio de baixa potência baseado } \\
\text { em identificação por radiofrequência (RFID) } \\
\text { passiva, o tricô na fabricação desse dispositivo, } \\
\text { onde o chip RFID foi costurado em um bolso } \\
\text { cercado por fio condutor, introduzido em uma } \\
\text { peça de vestuário elástica }\end{array}$ & (MONGAN et al. 2016) \\
\hline Sensor físico & Medida de glicose & $\begin{array}{l}\text { Sensores de fotopletismografia acoplados em } \\
\text { um revestimento opticamente sensível que } \\
\text { muda os parâmetros opticoquímicos }\end{array}$ & (RODIN et al., 2019) \\
\hline Sensor físico & $\begin{array}{l}\text { Medida da desidratação das } \\
\text { crianças }\end{array}$ & Sensores ópticos não invasivos & (VISSER et al., 2017) \\
\hline
\end{tabular}

Fonte: Construção dos autores. 
Nas técnicas não invasivas, uma das aplicações mais conhecidas é a medida da bioimpedância do tórax, que estima variação do volume cardíaco por meio da variação da impedância torácica (HENRIQUES, 2017). Para tal, posicionam-se quatro eletrodos em forma de fita, colocados em volta do pescoço e tórax, dois a dois, sendo esses últimos, posicionados um pouco abaixo do coração. Nos eletrodos mais externos aplica-se uma corrente e ao longo da medição da tensão nos eletrodos se obtém a variação de impedância. Esta técnica é baseada no fato de que a flutuação da quantidade de sangue nas cavidades cardíacas transforma a impedância da região ligada pelos eletrodos, por causa do crescimento de área da seção condutora e a dissemelhança de resistividade do sangue em relação aos outros tecidos (SLOMP, 1999). Hoje em dia, outras duas técnicas são comumente utilizados: o finapres (FINger Artetial PRESsure), que é não invasivo (GOMEZ; DIAN; ZEILER, 2020, JÄÄSKELÄINEN, 2018, TAPIA et al., 2017) e a tonometria de aplanação (ÖZCURA et al., 2017), que são procedimentos de aquisição da pressão arterial instantânea. Ambos se constituem em princípios similares, pois operam com uma onda de pressão, que é propagada pelas artérias para as bolsas externas que as circunda e estão infladas com ar.

A técnica finapres constitui-se, basicamente, de uma bolsa inflada com ar que envolve o dedo, onde o dedo é sujeitado a um pletismógrafo fotoelétrico (WALDRON; PATTERSON; JEFFRIES, 2018). Um servossistema pneumático de resposta rápida fica encarregado de manter o sinal elétrico do pletismógrafo igual a um valor pré-determinado. Por meio da variação da pressão na bolsa de ar, o valor de controle do pletismógrafo é geralmente regulado para que o volume da artéria envolvida fique sempre em dois terços do valor máximo. Em virtude da alteração de pressão na artéria, há uma tendência de oscilação do volume (aumentando e diminuído periodicamente), fazendo com que o sistema realimente o servossistema pneumático com a diferença entre a leitura do pletismógrafo e o valor de controle. Desta forma, a pressão interna da bolsa de ar que envolve o dedo acompanha a pressão intra-arterial, e é facilmente medida (SLOMP, 1999).

Já na tonometria de aplanação, o sistema é composto em um sensor de pressão de pequenas dimensões com uma alta sensibilidade (MEIDERT; SAUGEL, 2018), especificamente um micromanômetro piezo-resistivo, é posicionado sobre uma artéria superficial, geralmente a radial, femoral ou carótida. O sensor é então pressionado sobre a artéria diminuindo o seu calibre e a pressão interna da artéria é então transmitida transcutaneamente para o sensor, que registra a pressão (SLOMP, 1999). Recentemente, pesquisas tem integrado inteligência artificial a esse procedimento, com uso de redes neurais profundas (SPAIDE et al., 2020), o que tem sido uma tendência nos sensores utilizados nas aplicações biomédicas (AKSHAYA; SURESH; CARMEL, 2020; CHANDRA et al., 2019).

A oximetria de pulso também é outra técnica não invasiva, que serve para medir quanto de oxigênio o sangue arterial transporta (KIM et al., 2017; VERKRUYSSE et al.; 2017). Este sensor é formado por uma fonte de luz, composta por dois diodos emissores de luz (LEDs), e um fotodetector, colocado no lado oposto do sensor. O fotodetector recebe a luz originada pelos sensores e identifica a diferença 
entre a luz que foi absorvida pelas moléculas de hemoglobina (SANTANA, 2017). Esta aplicação vem ganhando atenção crescente nas ciências esportivas (PERREY; FERRARI, 2018; DI GIMINIANI et al, 2018) e pesquisas que permitam seu uso sem as limitações de baterias (ZHANG et al., 2019).

Atualmente, um nicho de pesquisa que vem se destacando e atraindo cada vez mais a atenção de pesquisadores e empresas são os sensores vestíveis (YAO; SWETHA; ZHU, 2018; HEIKENFELD et al., 2018). Estes sensores são dispositivos flexíveis que permitem um contato com a camada mais superficial da pele e trazem um monitoramento das condições de saúde das pessoas e desempenho físico (MAJUMDER; MONDAL; DEEN, 2017). Esses primeiros sensores que surgiram foram baseados para monitorar os sinais físicos, com temperatura da pele, pressão arterial, frequência respiratória e cardíaca. Porém, para englobar mais as condições de saúde da pessoa, foram criados sensores químicos e biossensores vestíveis que permitiram obter informações dos componentes químicos da epiderme (ZHANG et al., 2020). Outra recente aplicação são os sensores vestíveis epidérmicos, que apareceram com uma ordem de biossensores de tatuagem, aplicadas para detectar os níveis de lactato, glicose, álcool e ureia na transpiração humana (ZHANG et al., 2020). Zhang e colaboradores (2020), criaram um biossensor eletroquímico flexível baseados em nanofios de prata por impressão molecular para monitorar o lactato na transpiração durante a realização de exercícios físicos. Esse biossensor, que é uma célula de fluxo de polidimetilsiloxano, é implantado em um substrato flexível por um processo de serigrafia. Assim, a corrente de retorno do lactato é captada com medição na epiderme.

Promphet e colaboradores (2020), criaram um sensor colorimétrico vestível que é baseado em fios de algodão alterado para detectar de forma não invasiva e síncrona a glicose e ureia excretadas no suor humano. Das técnicas analíticas tradicionais, foi escolhido a colorimetria, devido a sua praticidade para o automonitoramento, simplicidade, fácil interpretação a olho nu e autodetectabilidade. Neste trabalho, os autores utilizaram nanofitas de celulose e óxido de quitosana-grafeno para modificar as superfícies do fio de algodão para melhorar a eficiência da imobilização enzimática e a atividade do sensor. Assim, esse dispositivo pode ser empregado nas roupas com facilidade ou diretamente na pele humana, onde destaca-se sua grande vantagem: não necessita de sangue para realizar a monitoração. (PROMPHET et al., 2020).

Já Mongan e colaboradores (2016), desenvolveram uma estrutura para os sensores biomédicos não invasivos, sem fio e vestível. Esta estrutura é usada para ajudar os pacientes mais jovens, especialmente os bebês prematuros, pois o monitoramento dos sinais vitais necessita de equipamentos que são fixados no corpo do paciente. Nas unidades neonatais, a fixação dos sensores é um problema recorrente, pois os prematuros têm uma área corporal bem pequena para posicionar todos os sensores, além do próprio desconforto imposto a eles. Essa solução vestível proposta surge como uma ferramenta de extrema importância nos dois aspectos: usabilidade e conforto. De modo geral, os autores criaram uma estrutura usando sensores sem fio de baixa potência baseado em identificação por radiofrequência (RFID) passiva. A técnica RFID requer um emissor e um receptor compatíveis 
para comunicação por radiofrequência (LOZANO-NIETO, 2017; LUO; GIL, FERNÁNDES-GARCÍA, 2020). Para tal, foi usado o tricô na fabricação desse dispositivo, onde o chip RFID foi costurado em um bolso cercado por fio condutor, introduzido em uma peça de vestuário elástica, para medir os sinais bioelétricos. Além disso, o circuito semicondutor e as placas de circuito impresso compactas foram alocadas de forma flexível na peça de vestuário. Para o monitoramento do biossinal, o conjunto RFID foi usado conjuntamente a um equipamento de chaveamento on-off de retrodifusão RFID (MONGAN et al., 2016). Outras aplicações biomédicas recentes e interessantes que utilizam RFID são (ZHANG et al., 2020; BHATTACHARYYA et al., 2018; CHMIELESKI; NOWOTARSKI, 2017).

Outra recente aplicação interessante foi realizada por Rodin e colaboradores (2019), que analisaram a confiabilidade e precisão da tecnologia de monitor não invasivo de glicose desenvolvido pela Spectrophon, Ltd (Israel), cujo sensor é composto de fotopletismógrafo. Essa técnica funciona iluminando a pele e avaliando as alterações na absorção de luz causadas pela dilatação e contração dos vasos sanguíneos. Para al, esse dispositivo utiliza sensores de fotopletismografia acoplados em um revestimento opticamente sensível que muda os parâmetros opticoquímicos na presença de compostos específicos no suor. Assim, eles são analisados em um algoritmo dedicado a derivar as concentrações de glicose no sangue. Para comunicação, o biossensor foi acoplado em um smartwatch, o qual possui o elemento óptico necessário para fotopletismografia. Neste ponto ressalta-se que diversas outras pesquisas têm utilizados dispositivos inteligentes do dia-a-dia como smatphones e smartwatches integrados a aplicações biomédicas. O uso desses dispositivos permite acesso rápido e fácil a informação, comunicação integrada entre paciente e médico, com acesso remoto e compartilhamento de dados, entre outras vantagens. Alguns exemplos podem ser vistos em (PUROHIT et al., 2020; ZHAO et al., 2020; CEYLAN, H, K.; OZCAN, 2018; GUO, 2017; GAN; POON, 2016, LEE, 2016), frequentemente associados a rotinas de inteligência artificial (DZOBO et al., 2020; BANERJEE et al., 2020; SHAHMOHAMMADI et al., 2017; WEISS et al., 2016).

Por fim, outro trabalho de destaque foi realizado por Visser e colaboradores (2017), onde analisaram quatro sensores ópticos não invasivos diferentes para avaliar a desidratação das crianças, baseados na medição quantitativa e objetiva de vários marcadores clínicos de desidratação. Os quatro sensores foram analisados para determinar a capacidade de avaliação quantitativa da intensidade da desidratação. Os quatro aspectos testados são: tempo de recarga capilar, tempo de recuo da pele, perfil de temperatura da pele e espectrometria de infravermelho. Foram realizados experimentos com dez pacientes e observado que para cada grupo há uma fusão sensorial mais adequada, porém, em geral, os sensores que baseiam-se no perfil de temperatura e tempo de recuo da pele apresentaram mais sensibilidade. 


\section{SENSORES INVASIVOS E SUAS APLICAÇÕES BIOMÉDICAS}

Diferentemente dos sensores não invasivos, nos métodos invasivos é necessária alguma intervenção cirúrgica para posicionamento do sensor, mesmo que minimamente. Um exemplo simples são os cateteres utilizados para medir a pressão arterial, comparando-a a uma pressão de referência, em uma dispositivo chamado de bolsa de pressão, conforme esquema mostrado na Figura 2.

Figura 2 - Esquema de medição de pressão por meio invasivo.

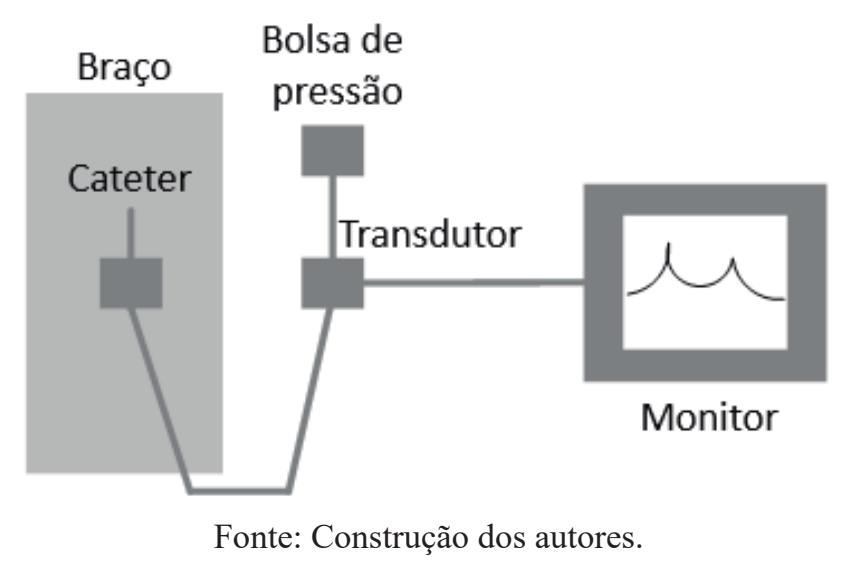

Um exemplo típico é o cateter de condutância, que mede o volume de cardíaco instantâneo (FELDMAN et al., 2016; FELDMAN et al., 2017). Esta técnica introduz, na cavidade cardíaca a ser medida, um cateter com eletrodos que ficam dispostos uniformemente ao longo do seu eixo longitudinal e faz propagar uma menor corrente entre os eletrodos dos extremos. Pela medição das tensões geradas nos diversos eletrodos, que variam com o volume de sangue que os envolve, pode-se avaliar a condutância de cada segmento, aproximá-los por cilindros e deste modo estimar o volume instantâneo da cavidade cardíaca. Sendo que a área por onde a corrente flui é maior, e aumenta também a condutância (SLOMP, 1999). Recentemente, estudos mostraram que há modelos matemáticos precisos para estimar a área e o diâmetro das artérias, que são validados através de técnicas de variação de frequências e optocaptação (DABRI; KASSAB, 2018).

$\mathrm{Na}$ aquisição da pressão arterial, os métodos invasivos constituem em cateteres inseridos em artérias, normalmente na artéria radial, mas podem ser outras artérias como femoral, dorsal do pé, braquial e artérias axilares. As artérias são canuladas percutaneamente ou algumas vezes através de uma incisão. Estes cateteres utilizam como sensores de pressão micromanômetros e pontes de extensômetros resistivos, os strain-gauges, inseridos na membrana elásticas (SLOMP, 1999). Para tal, os micromanômetros são fixados na ponta dos cateteres e são de fato introduzidos nas artérias do paciente e prosseguem até ponto de interesse. Nas pontes de strain-gauges, a pressão arterial é transmitida por meio do cateter tubular, que a atua como um vaso comunicante. $\mathrm{O}$ cateter fica preenchido com soro e uma de suas extremidades é aberta, onde é posicionada na cavidade cardíaca ou na artéria onde será medida a 
pressão. Assim, o sensor extensométrico se encontra na cavidade externa (SLOOMP, 1999). Hoje em dia, já há métodos não invasivos para medição da pressão arterial (ROGGE et al., 2019), bem como métodos indiretos baseados na pressão arterial pulmonar (GREENHUT, 2016).

Mohan e colaboradores (2017), desenvolveram um biossensor minimamente invasivo, baseado em microagulhas, para monitorar, em tempo real, o nível de álcool do fluído intersticial. Essas microagulhas tem tamanho de um mícron, no qual consegue romper a camada externa pele para alcançar o fluído intersticial com um nível de dor e danos muito baixos. Foi construído um sistema de três eletrodos de microagulhas piramidais por meio da junção de fios de platina e prata. Na abertura das microagulhas, os fios são cuidadosamente posicionados, em lugares específicos no lúmen da microagulha, com o intuito de construir o transdutor eletroquímico na abertura da microagulha. Para a avaliar o nível de álcool do fluído intersticial e a capacidade do método das microagulhas adentrar na pele, os autores realizaram testes na pele de camundongo morto. Diversas outras pesquisas tem o foco de reduzir o tamanho dos biossensores, pois com essa miniaturização, há uma considerável alivio do desconforto causado pelo sensor invasivo, vide alguns exemplos de microbiossensores recentemente desenvolvidos (GANESANA et al., 2019; BUK; PEMBLE, 2019; HOSSAIN et al., 2018; IN et al., 2019; ZHAO et al., 2017). Existem outro sensores invasivos que são construídos de forma integrada aos seus atuadores, como por exemplo: marca-passos implantáveis (ELIAV et al., 2020), cardioversores-desfibriladores implantáveis (GOLWALA et al., 2017; QUAST et al., 2019) e implantes cocleares (DHANASINGH; JOLLY, 2017, LENARZ, 2017), entre outros. Nesses dispositivos, os objetivos são comuns: aumentar o tempo de vida das baterias, reduzir o tamanho do dispositivo para fornecer maior conforto ao paciente e minimizar a quantidade de fios na aquisição das grandezas de interesse, tradicionalmente mensuradas por eletrodos, além de aumentar o desempenho e dispor de novas funções, tais como a comunicação wireless. Como esses sensores são integrados aos dispositivos atuadores, não serão abordados em com mais detalhes nesse trabalho, pois tais dispositivos merecem um estudo a parte, devido as suas complexidades e quantidade de inovações que vem sendo proposta nos últimos anos.

\section{DISCUSSÕES}

Segundo estudos, um dos métodos invasivos para aquisição de pressão arterial que pode trazer complicações é o cateterismo, sendo a trombose a mais comum delas, sendo que esse risco aumenta com expansão do tamanho do cateter e a duração da cateterização. O uso deste método também pode causar embolização, infecção, neuropatia e pseudoaneurisma arterial (SLOMP, 1999). Assim, a perspectiva para essa aplicação biomédica é que os novos sensores sejam cada vez menores, utilizando de tecnologias modernas como as utilizadas na fabricação de microbiossensores.

Já na tonometria de aplanação, a maioria dos estudos realizados relatam que o sinal adquirido é uma média de vários batimentos selecionados (SLOMP, 1999). Isso faz a forma de onda adquirida 
ser mais precisa, mas impede sua correlação com o volume ventricular. Outra desvantagem é que esse método é muito sensível aos movimentos e posicionamento do sensor. Logo, há uma tendência para o uso de sensores vestíveis, bem como a fusão sensorial de uma rede de sensores que garanta a correta correlação das grandezas medidas individualmente.

Imholz e coloboradores (1998) mostraram que o método finapres, em comparação com as técnicas não invasivas, expressa pressões sistólicas estatisticamente maiores, porém não são clinicamente relevantes. Além do mais, pelo equipamento ser conectado ao dedo da mão, precisa de uma relativa estabilidade, pois a medição da pressão torna-se praticamente impossível em diversos movimentos. Assim, permite somente a execução de exercícios para os membros inferiores e o membro superior contralateral. Essa restrição, pode ser concedida ao método invasivo, uma vez que a cateterização da artéria impede o movimento do membro utilizado na medida (POLITO; FERINATTI, 2003). Portanto, novamente, os sensores vestíveis, minimamente invasivos e biossensores destacam-se como frentes de auspiciosa viabilidade, principalmente na área esportiva, onde a bioengenharia tem contribuído para análise de desempenho de atletas e monitoramento contínuo durante a execução dos exercícios físicos.

Já na oximetria de pulso, quando foi testado um novo equipamento, constatou-se que as leituras de saturação de oxigênio estabilizaram após dois minutos com dispositivo devidamente posicionado. Apesar de que, na prática clínica, as leituras são gravadas, geralmente, de forma consecutiva. Contudo, oximetria de pulso não mede a pressão arterial de dióxido de carbono, e não é capaz de informar sobre a ventilação do paciente (DOMINGO et al., 2010). Outro ponto importante de citar é que a calibração do oxímetro deve ser feita uma vez por ano ou quando necessário, para que este seja sempre preciso, mesmo que o aparelho já faça um auto-calibração antes de iniciar. Assim, há uma tendência ao uso de algoritmos de aprendizagem, tais como redes neuras artificiais profundas, para que mantenha o dispositivo nos padrões desejados. Entretanto, com tecnologias inteligentes e de auto-organização, surgem os desafios de custo computacional para execução desses algoritmos em microprocessadores mais simples, devido as limitações de hardware. Outro ponto que deve ser levado em consideração é o uso das baterias que esse processamento contínuo consumirá. Assim, há também um nicho para pesquisa de baterias autorregenerativas e baseadas em supercapacitores.

Ainda, o biossensor eletroquímico baseado em nanofios de prata impressos molecularmente e a rede de nanofios de prata revestida com eletrodo de carbono serigrafado mostraran excelente condutividade e estabilidade elétrica. As impressões moleculares de lactato no eletrodo de carbono apresentaram alta sensibilidade e seletividade para a detecção do lactato no suor. Portanto esse biossensor se mostrou como uma técnica excelente para monitorar de forma não invasiva as substâncias metabólicas do suor. Essa monitoração contribui para avaliação das condições fisiológicas, sendo assim, uma ferramenta de avaliação muito interessante para a área esportiva, principalmente para atletas que fazem atividades com elevado uso de força e resistência (ZHANG et al., 2020). Conforme discutido anteriormente, a área dos esportes tem utilizado cada vez mais os recursos biotecnológicos 
para análise de desempenho e resistência dos atletas, sendo uma área em franca expansão, o que a torna um terreno fértil para novas aplicações e tecnologias.

Outra nova tecnologia de grande importância é o sensor vestível a base de algodão, utilizado para a detecção de glicose e ureia no suor. Ele consegue detectar a glicose em um intervalo de 0,1 a 3 mM com resolução de $0,1 \mathrm{mM}$, e a ureia entre 30 a $180 \mathrm{mM}$ com resolução de $30 \mathrm{mM}$ (PROMPHET et al., 2020). As vantagens dos sensores vestíveis em relação aos sensores tradicionais são várias, tais como: flexibilidade, usabilidade, ausência de fios, conforto, entre outras. Entretanto, há diversos desafios que devem ser levados em consideração no projeto de um sensor vestível, tais como: estabilidade, robustez e comunicação com a interface de monitoramento.

Em se tratando de monitoramento do paciente, os sensores de tecido de malha usando o RFID cumprem essa função com bons resultados, isto é, fornecem biofeedbacks de forma não invasiva e confortável ao paciente, pois o RFID é uma tecnologia que usa ondas de rádio para identificar pessoas ou objetos, ou seja, não há contato direto com o paciente monitorado (ROBERTS, 2005). Entretanto, os desafios referentes aos sensores vestíveis se mantêm, além de incluir mais alguns, tais como a fabricação da antena RFID miniaturizada, processamento de sinal de baixa amplitude sujeito a ruídos exógenos, rejeição de distúrbios e interferência na coleta dos dados, bem como o armazenamento desses dados de forma segura. Assim, a Ciência de Dados e a Computação passam a contribuir na superação das barreiras para as novas tecnologias biomédicas, pois a segurança dos dados torna-se fundamental na era atual, bem como formas seguras de armazenamento e transferência de dados sem fio.

Além dessas aplicações, o novo biossensor desenvolvido para a avaliar o nível de glicose baseado em fotopletismografia mostrou ser uma ferramenta com alto potencial. Esse tipo de dispositivo está sendo desenvolvido para trazer uma conformidade ao paciente e oferecer uma ferramenta de fácil acessibilidade, permitindo que o paciente monitore o nível de glicose de forma instantânea, além de fornecer um histórico para a avalição (RODIN et al., 2019). Nesse ponto tem-se outra tendência das aplicações biomédicas, a integração com os dispositivos inteligentes, como smartphones e smartwatches, pois permitem um acesso rápido e fácil. Além disso, desta forma, é possível que o paciente compartilhe informações de monitoramento com seu médico através de comunicação via internet, ou ainda, o dispositivo inteligente pode disparar mensagens de alerta ao médico de forma periódica ou por gatilhos emergenciais. Assim, tem-se uma tendência ao desenvolvimento de dispositivos biomédicos integrando IoT (Internet of Things) em um futuro breve. Pode-se também considerar que esses novos dispositivos possuirão inteligência artificial, o que novamente recai aos desafios de custo computacional para execução e viabilidade de uso dessa abordagem devido as baterias. Logo, surge a perspectiva da execução desses algoritmos inteligentes em servidores remotos e não mais nos dispositivos em posse dos usuários. Entretanto, haverá o requerimento de conexão constante e estável com internet, o que para muitas localidades, ainda é um desafio a ser superado. 


\section{CONCLUSÃO}

Conforme pode ser analisado ao longo das aplicações discutidas, os sensores invasivos provocam desconforto e podem, inclusive, causar complicações a saúde ao paciente. Assim, sempre que possível são evitados, pois é necessária internação e cirurgia para realizar o procedimento de inserção do sensor. Nos casos em que se faz obrigatório o uso de sensor invasivo, opta-se por projetá-lo de forma minimamente invasiva, preferencialmente sem fio ou com tecnologias de micro ou nanobiossensores. Ainda, devido a constante evolução das tecnologias na área da saúde e a necessidade de promover ao máximo a segurança e bem-estar do paciente, a tendência é a utilização de sensores não invasivos do tipo vestíveis, uma tecnologia que está avançando rapidamente e com sucesso nos resultados almejados. Outro ponto discutido foi a monitorização dos pacientes, onde as comunicações wireless possui vantagens de grande valia em comparação a comunicação com fio, tais como: maior estabilidade do sensor pela própria inexistência do cabo, facilidade de mobilidade do paciente dentro do hospital e a viabilidade de realizar a monitorização com ou sem um monitor multiparamétrico hospitalar, por meio de aplicativos, auxiliando na definição do diagnóstico e no acompanhamento dos pacientes, sendo possível supervisionar os dados do paciente de qualquer lugar com acesso à internet em um tablet ou smartphone.

\section{REFERÊNCIAS}

AKSHAYA, T J H; SURESH, V.; CARMEL, S. M. Transforming Biomedical Applications Through Smart Sensing and Artificial Intelligence. In: Deep Neural Networks for Multimodal Imaging and Biomedical Applications. IGI Global, 2020. p. 186-204. Disponível em: https://bit.ly/3mEOJUP

BANERJEE, A. CHAKRABORTY, C.; KUMAR, A.; BISWAS, D. Emerging trends in IoT and big data analytics for biomedical and health care technologies. In: Handbook of data science approaches for biomedical engineering. Academic Press, 2020. p. 121-152. Disponível em: https://bit.ly/37AwtFM

BANERJEE, H.; SHEN, S.; REN, H. Magnetically actuated minimally invasive microbots for biomedical applications. In: Electromagnetic Actuation and Sensing in Medical Robotics. Springer, Singapore, 2018. p. 11-41. Disponível em: https://bit.ly/2L4InjT

BHATTACHARYYA, M.; GRUENWALD, W.; JANSEN, D.; REINDL, L.; AGHASSI-HAGMANN, J. Design of a programmable passive SoC for biomedical applications using RFID ISO 15693/NFC5 interface. Journal of Low Power Electronics and Applications, v. 8, n. 1, p. 3, 2018. Disponível em: https://bit.ly/3qpCJZP 
BRONZINO, J. D. Medical devices and systems. CRC Press, 2006. Disponível em: https://bit.ly/ 2L6exvp

BUK, V.; PEMBLE, M. E. A highly sensitive glucose biosensor based on a micro disk array electrode design modified with carbon quantum dots and gold nanoparticles. Electrochimica Acta, v. 298, p. 97-105, 2019. Disponível em: https://bit.ly/3g4L9B3

BURRITT, M. F. Noninvasive and invasive sensors for patient monitoring. Laboratory Medicine, v. 29, n. 11, p. 684-687, 1998. Disponível em: https://bit.ly/39Run7B

CEYLAN, H, K.; OZCAN, A. Smartphones democratize advanced biomedical instruments and foster innovation. Clinical Pharmacology \& Therapeutics, v. 104, n. 1, p. 38-41, 2018. Disponível em: https://bit.ly/31Cz730

CHANDRA, S.; GUPTA, R.; GHOSH, S.; MONDAL, S. An intelligent and power efficient biomedical sensor node for wireless cardiovascular health monitoring. IETE Journal of Research, p. 1-11, 2019. Disponível em: https://bit.ly/3g6uOfl

CHMIELEWSKI, M.; NOWOTARSKI, M. Sensor-based supporting mobile system Parkinson disease clinical tests utilising biomedical and RFID technologies. In: MATEC Web of Conferences. EDP Sciences, 2017. p. 05003. Disponível em: https://bit.ly/2JAXGAe

CHINNADAYYALA, S.; R.; PARK; K. D.; CHO, S. Review - in vivo and in vitro microneedle based enzymatic and non-enzymatic continuous glucose monitoring biosensors. ECS Journal of Solid State Science and Technology, v. 7, n. 7, p. Q3159, 2018. Disponível em: https://bit.ly/36BWohd

DABIRI, A. E.; KASSAB, G. S. Injection-less conductance method for vascular sizing. Frontiers in physiology, v. 9, p. 371, 2018. Disponível em: https://bit.ly/3mCAxfl

DHANASINGH, A.; JOLLY, C. An overview of cochlear implant electrode array designs. Hearing research, v. 356, p. 93-103, 2017. Disponível em: https://bit.ly/3mxH22R

DI GIMINIANI, R.; LANCIA, S.; FERRARI, M.; QUARESIMA, V.; VISTISEN, H. T.; KLILTGAARD, A.; HEICK, R. A.; OESTERGARD, K.; SOERENSEN, K. Y,. CARDINALE, M. A wearable integrated textile EMG and muscle oximetry system for monitoring exercise-induced effects: a feasibility study. In: 2018 IEEE International Symposium on Medical Measurements and Applications (MeMeA). IEEE, 2018. p. 1-5. Disponível em: https://bit.ly/3oj2Mjs 
DOMINGO, C.; BLANCH, L.; MURIAS, G.; LUJÁN, M. State-of-the-art sensor technology in Spain: Invasive and non-invasive techniques for monitoring respiratory variables. Sensors, v. 10, n. 5, p. 4655-4674, 2010. Disponível em: https://bit.ly/39FTUjF

DORF, Richard C. Sensors, Nanoscience, Biomedical Engineering, and Instruments: Sensors Nanoscience Biomedical Engineering. CRC press, 2018. Dsponível em: https://bit.ly/33EYwTz

DZOBO, K.. ADOTEY, S.; THOMFORD, N. E.; DZOBO, W. Integrating artificial and human intelligence: a partnership for responsible innovation in biomedical engineering and medicine. OMICS: A Journal of Integrative Biology, v. 24, n. 5, p. 247-263, 2020. Disponível em: https://bit.ly/3oobXPs

ELIAV, R., ELITZUR, Y., PLANER, D., BEERI, R., GILON, D., SHUVY, M.; LURIA, D.; DANENBERG, H.; PERLMAN, G. Y. Predictors for permanent pacemaker implantation following transcatheter aortic valve implantation: trends over the past decade. Journal of Interventional Cardiac Electrophysiology, p. 1-9, 2020. Disponível em: https://bit.ly/3g5Gpel

FELDMAN, M. D.; Valvano, J. W.; Pearce, J. A.; Wei, C. L. Method and apparatus for determining cardiac performance in a patient with a conductance catheter. U.S. Patent n. 9,380,946, 5 jul. 2016. Disponível em: https://patents.google.com/patent/US9380946B2/en

FELDMAN, M. D.; VALVANO, J. W.; PEARCE, J. A.; WEI, C. L. Method and apparatus for determining cardiac performance in a patient with a conductance catheter. U.S. Patent n. 9,820,674, 21 nov. 2017. Disponível em: https://patents.google.com/patent/US9820674B2/en

GOLWALA, H.; BAJAJ. N. S.; ARORA, G.; ARORA, P. Implantable cardioverter-defibrillator for nonischemic cardiomyopathy: an updated meta-analysis. Circulation, v. 135, n. 2, p. 201-203, 2017. Disponível em: https://bit.ly/3lwMSAc

GAN, S. K.; POON, J. The world of biomedical apps: their uses, limitations, and potential. Scientific Phone Apps and Mobile Devices, v. 2, n. 1, p. 6, 2016. Disponível em: https://bit.ly/2L8wB8h

GANESANA, M., TRIKANTZOPOULOS; E., MANIAR, Y.; LEE, S. T.; VENTON, B. J. Development of a novel micro biosensor for in vivo monitoring of glutamate release in the brain. Biosensors and Bioelectronics, v. 130, p. 103-109, 2019. Disponível em: https://bit.ly/2KW7t4e 
GOMEZ, A.; DIAN, J.; ZEILER, F. A. Continuous and entirely non-invasive method for cerebrovascular reactivity assessment: technique and implications. Journal of Clinical Monitoring and Computing, p. 1-9, 2020. Disponível em: https://bit.ly/33G8PXE

GREENHUT, S. E. Measurement of cardiac cycle length and pressure metrics from pulmonary arterial pressure. U.S. Patent n. 9,314,205, 19 abr. 2016. Disponível em: https://bit.ly/3mEobmH

GUO, J. Smartphone-powered electrochemical biosensing dongle for emerging medical IoTs application. IEEE Transactions on Industrial Informatics, v. 14, n. 6, p. 2592-2597, 2017. Disponível em: https:// bit.ly/3qp5DJx

HEIKENFELD, J., JAJACK, A., ROGERS, J., GUTRUF, P., TIAN, L., PAN, T., LI, R.; KHINE, M.; KIM, J.; WANG, J.; KIM, J. WEARABLE sensors: modalities, challenges, and prospects. Lab on a Chip, v. 18, n. 2, p. 217-248, 2018. Disponível em: https://rsc.li/3qpMTtr

HENRIQUES, M. S. Bioimpedância Torácica: Aplicação da hemodinâmica não-invasiva no tratamento da hipertensão. 2017. Tese (Doutorado) - Universidade da Beira Interior, Portugal. Disponível em: https://bit.ly/2JGcbTk

HOSSAIN, I.; TAN, C.; DOUGHTY, P. T.; DUTTA, G.; MURRAY, T. A.; SIDDIQUI, S.; IASEMIDIS, L.; ARUMUGAM, P. U. A novel microbiosensor microarray for continuous ex vivo monitoring of gamma-aminobutyric acid in real-time. Frontiers in neuroscience, v. 12, p. 500, 2018. Disponível em: https://bit.ly/2IbFqNo

IMHOLZ B. P.; WIELING W.; VAN MONTFRANS G. A; WESSELING, K. H. Fifteen years experience with finger arterial pressure monitoring: assessment of the technology. Cardiovasc Res 1998; 38:605-16. Disponível em: https://bit.ly/3qrBYQ0

KOIZUMI, M. T. Desenvolvimento de sensores e instrumentação aplicados no projeto de equipamentos analisadores de qualidade de energia elétrica. Dissertação (Mestrado) - Universidade Tecnológica Federal do Paraná. 2012. Disponível em: https://bit.ly/2KWTpYg

JÄÄSKELÄINEN, L. Cuffless and noninvasive method for pulse transit time based continuous blood pressure estimation. 2018. Tese (Doutorado), Aalto University. Disponível em: https://bit.ly/37Bgs2J 
KIM, J.; GUTRUF, P.; CHIARELli, A. M.; HEO, S.; CHO, K.; XIE, Z.; BANKS, A.; HAN, S.; JANG, K.; LEE, K.; FENG, X.; HUANG, Y.; FABIANI, M.; GRATTONm G.; PAIK, U.; ROGERS, J. A.. Miniaturized battery-free wireless systems for wearable pulse oximetry. Advanced functional materials, v. 27, n. 1, p. 1604373, 2017. Disponível em: https://bit.ly/2L0Z1Rj

LAI, J.; YI, Y.; ZHU, P.; SHEN, J.; WU, K.; ZHANG, L.; LIU, J. Polyaniline-based glucose biosensor: A review. Journal of Electroanalytical Chemistry, v. 782, p. 138-153, 2016. Disponível em: https:// bit.ly/39Eo3QH

LENARZ, T. Cochlear implant-state of the art. Current Topics in Otorhinolaryngology, Head and Neck Surgery, v. 16, p. 1-29, 2017. Disponível em: https://bit.ly/3g4VLQp

LOUZADA, D. R.; MONTEIRO, E. C.; GUSMÃO, L. A. P.; BARBOSA, C. H. Medição não-invasiva de ondas de pulso arterial utilizando transdutor de pressão MIG. In: IV Latin American Congress on Biomedical Engineering 2007, Bioengineering Solutions for Latin America Health. Springer, Berlin, Heidelberg, 2007. p. 436-439. Disponível em: https://bit.ly/2JNdn76

LOZANO-NIETO, Albert. RFID design fundamentals and applications. CRC press, 2017. Disponível em: https://bit.ly/3olo4wV

LUO, Chengyang; GIL, Ignacio; FERNÁNDEZ-GARCÍA, Raúl. Wearable textile UHF-RFID sensors: A systematic review. Materials, v. 13, n. 15, p. 3292, 2020. Disponível em: https://bit.ly/2JqNuum

MAJUMDER, S.; MONDAL, T.; DEEN, M. Jamal. Wearable sensors for remote health monitoring. Sensors, v. 17, n. 1, p. 130, 2017. Disponível em: https://bit.ly/2L6pddt

MEIDERT, Agnes S.; SAUGEL, Bernd. Techniques for non-invasive monitoring of arterial blood pressure. Frontiers in medicine, v. 4, p. 231, 2018. Disponível em: https://bit.ly/3g4e2x6

MOHAN, A. V.; WINDMILLER, J. R.; MISHRA, R. K.; WANG, J. Continuous minimally-invasive alcohol monitoring using microneedle sensor arrays. Biosensors and Bioelectronics, v. 91, p. 574-579, 2017. Disponível em: https://bit.ly/37q2LDm 
MONGAN, W.; ANDAY, E.; DION, G.; FONTECCHIO, A.; JOYCE, K.; KURZEG, T.; LIU, Y.; MONTGOMERY, O.; RASHEED, I.; SAHIN, C.; VORA, S.; DANDEKAR, K. A multi-disciplinary framework for continuous biomedical monitoring using low-power passive RFID-based wireless wearable sensors. In: 2016 IEEE International Conference on Smart Computing (SMARTCOMP). IEEE, 2016. p. 1-6. Disponivel em: https://bit.ly/2I6NWwY

ÖZCURA, F.; YILDIRIM, N.; TAMBOVA, E.; SAHIN, A. Evaluation of Goldmann applanation tonometry, rebound tonometry and dynamic contour tonometry in keratoconus. Journal of optometry, v. 10, n. 2, p. 117-122, 2017. Disponível em: https://bit.ly/3qxQ1T1

PERREY, S.; FERRARI, M. Muscle oximetry in sports science: a systematic review. Sports Medicine, v. 48, n. 3, p. 597-616, 2018. Disponível em: https://bit.ly/31CL31k

POLITO, M. D.; FARINATTI, P. T. Vs. Considerações sobre a medida da pressão arterial em exercícios contra-resistência. Revista Brasileira de Medicina do Esporte, v. 9, n. 1, p. 25-33, 2003. Disponível em: https://bit.ly/31L4tol

PROMPHET, N.; HINESTROZA, J. P.; RATTANAWALEEDIROJIN, P.; SOATTHIYANON, N.; SIRALERTMUKUL, K.; POTIYARAJJ,P.; RODTHONGKUM, N. Cotton thread-based wearable sensor for non-invasive simultaneous diagnosis of diabetes and kidney failure. Sensors and Actuators B: Chemical, p. 128549, 2020. Disponível em: https://bit.ly/2VxG0rF

PRUTCHI, D.; NORRIS, M. Design and development of medical electronic instrumentation: a practical perspective of the design, construction, and test of medical devices. John Wiley \& Sons, 2005. Disponível em: https://bit.ly/2I6ORxq

PUROHIT, B.; KUMAR, A.; MAHATO, K.; CHANDRA, P. Smartphone-assisted personalized diagnostic devices and wearable sensors. Current Opinion in Biomedical Engineering, v. 13, p. 42-50, 2020. Disponível em: https://bit.ly/2VyamKF

QUAST, A. F. B.; BAALMAN, S. W.; BROUWER, T. F.; SMEDING, L.; WILDE, A. A.; BURKE, M. C.; KNOPS, R. E. A novel tool to evaluate the implant position and predict defibrillation success of the subcutaneous implantable cardioverter-defibrillator: the PRAETORIAN score. Heart rhythm, v. 16, n. 3, p. 403-410, 2019. Disponível em: https://bit.ly/3qoBLgr 
RAY, P. P. Continuous glucose monitoring: a systematic review of sensor systems and prospects. Sensor Review, v. 38, n. 4, p. 420-437, 2018. Disponível em: https://bit.ly/2VA7dKw

ROBERTS, C. M. Radio frequency identification (RFID). Computers \& security, v. 25, n. 1, p. 18-26, 2006. Disponível em: https://bit.ly/39CRKRU

RODIN, D.; KIRBYY, M.; SEDOGIN, N.; SHAPIRO, Y.; PINHASOV, A.; FREININ, a. Comparative accuracy of optical sensor-based wearable system for non-invasive measurement of blood glucose concentration. Clinical biochemistry, v. 65, p. 15-20, 2019. Disponível em: https://bit.ly/39GeMHI

ROGGE, D. E.; NICKLAS, J. Y.; SCHÖN, G.; GROTHE, O.; HAAS, S. A.; REUTER, D. A.; SAUGEL, B. Continuous noninvasive arterial pressure monitoring in obese patients during bariatric surgery: an evaluation of the vascular unloading technique (Clearsight system). Anesthesia $\&$ Analgesia, v. 128, n. 3, p. 477-483, 2019. Disponível em: https://bit.ly/2VyaXfn

ROOINTAN, A.; MIR, T. A., WANI, S. I., HUSSAIN, K. K., AHMED, B., ABRAHIM, S., SAVARDASHTAKI, A.; GANDOMANI, G.; GANDOMANI, G.; CHINNAPPAN, R.; AKHTAR, M. H. Early detection of lung cancer biomarkers through biosensor technology: A review. Journal of pharmaceutical and biomedical analysis, v. 164, p. 93-103, 2019. Disponível em: https://bit.ly/ 37z6uyC

SANTANA, Isabela Souto. Desenvolvimento de uma placa didática de um oxímetro de pulso. Disponível em: https://bit.ly/2VxYdFB.

SHAHMOHAMMADI, F.; HOSSEINI, A.; KING, C. E.; SARRAFZADEH, M. Smartwatch based activity recognition using active learning. In: 2017 IEEE/ACM International Conference on Connected Health: Applications, Systems and Engineering Technologies (CHASE). IEEE, 2017. p. 321-329. Disponível em: https://bit.ly/33IK1Oq

SHI, S.; ANG, E. L.; ZHAO, H. In vivo biosensors: mechanisms, development, and applications. Journal of industrial microbiology \& biotechnology, v. 45, n. 7, p. 491-516, 2018. Disponível em: https://bit.ly/2VBYVS4

SLOMP, M. Sistema de Aquisição do Ciclo Pressão-Volume Cardíaco por Métodos Não-Invasivos. Dissertação (Mestrado em Engenharia Elétrica) - Escola de Engenharia, Universidade Federal do Rio Grande do Sul. Porto Alegre. 1999. Disponível em: https://bit.ly/36A7vXW 
SPAIDE, T.; WU, Y.; YANAGIHARA, R. T.; FENG, S.; GHABRA, O.; JONATHAN, S. Y.; CHEN, P. P.; MOSES, F.; LEE, A. Y.; WEN, J. C. Using Deep Learning to Automate Goldmann Applanation Tonometry Readings. Ophthalmology, 2020. (In press). Disponível em: https://bit.ly/31GON1M

TAPIA, G.; SALINAS, M.; PLAZA, J.; MELLADO, D.; SALAS, R.; SAAVEDRA, C.; VELOZ, A.; ARRIOLA, A.; IDIAQUEZ, J.; GLARÍA, A. Photoplethysmogram Fits Finger Blood Pressure Waveform for non-Invasive and minimally-Intrusive Technologies-Evaluation of Derivative Approaches. In: International Conference on Bio-inspired Systems and Signal Processing. ScitePress, 2017. p. 155-162. Disponível em: https://bit.ly/36zcZ51

TAYLOR, R. M.; MILLER, P. R.; EBRAHIMI, P.; POLSKY, R.; BACA, J. T. Minimally-invasive, microneedle-array extraction of interstitial fluid for comprehensive biomedical applications: transcriptomics, proteomics, metabolomics, exosome research, and biomarker identification. Laboratory animals, v. 52, n. 5, p. 526-530, 2018. Disponível em: https://bit.ly/37sySm2

TOGAWA, Tatsuo; TAMURA Toshiyo; OBERG, P. Ake. Biomedical Sensors and Instruments. Second Edition, CRC Press, 2011.

VERKRUYSSE, W.; BARTULA, M.; BRESCH, E.; ROCQUE, M.; MEFTAH, M.; KIRENKO, I. Calibration of contactless pulse oximetry. Anesthesia and analgesia, v. 124, n. 1, p. 136, 2017. Disponível em: https://bit.ly/3qpp980

VISSER, C.; KISSER, E,; DELLIMORE, K.; VAN DER HEEVER, D.; SMITH, J. Investigation of the feasibility of non-invasive optical sensors for the quantitative assessment of dehydration. Medical Engineering \& Physics, v. 48, p. 181-187, 2017. Disponível em: https://bit.ly/2Jx4Fu9

WANG, P.; LIU, Q. Biomedical sensors and measurement. Springer Science \& Business Media, 2011. Disponível em: https://bit.ly/33Cm2R2

WALDRON, M.; PATTERSON, S. D.; JEFFRIES, O. Inter-day reliability of Finapres ${ }^{\circledR}$ cardiovascular measurements during rest and exercise. Sports medicine international open, v. 2, n. 1, p. E9, 2018. Disponível em: https://bit.ly/2VxVwE1

WEBSTER, J. G. Medical instrumentation: application and design. John Wiley \& Sons, 2009. Disponível em: https://bit.ly/36DYddI 
WEISS, G. M.; TIMKO, J. L.; GALLAGHER, C. M.; YONEDA, K.; SCHREIBER, A. J. Smartwatch-based activity recognition: A machine learning approach. In: 2016 IEEE-EMBS International Conference on Biomedical and Health Informatics (BHI). IEEE, 2016. p. 426-429. Disponível em: https:// bit.ly/36DoNmU

XIAO, T.; WU, F.; HAO, J.; ZHANG, M.; YU, P.; MAO, L. In vivo analysis with electrochemical sensors and biosensors. Analytical Chemistry, v. 89, n. 1, p. 300-313, 2017. Disponível em: https:// bit.ly/3qoDxy7

XUE, P.; ZHANG, L.; XU, Z.; YAN, J.; GU, Z.; KANG, Y. Blood sampling using microneedles as a minimally invasive platform for biomedical diagnostics. Applied Materials Today, v. 13, p. 144-157, 2018. Disponível em: https://bit.ly/36C0xlq

YAO, S.; SWETHA, P.; ZHU, Y. Nanomaterial-Enabled wearable sensors for healthcare. Advanced healthcare materials, v. 7, n. 1, p. 1700889, 2018. Disponível em: https://bit.ly/39zmL9w

YIN, M. J.; GU, B.; AN, Q. F.; YANG, C.; GUAN, Y. L.; YONG, K. T. Recent development of fiber-optic chemical sensors and biosensors: Mechanisms, materials, micro/nano-fabrications and applications. Coordination Chemistry Reviews, v. 376, p. 348-392, 2018. Disponível em: https://bit.ly/2VB0eki

ZHANG, H.; GUTRUF, P.; MEACHAM, K.; MONTANA, M. C.; ZHAO, X.; CHIARELLI, A. M.; VAZQUEZ-GUARDADO, A.; NORRIS, A.; LU, A.; GUO, Q.; XU, C.; WU, .; ZHAO, X.; BAI, W.; KANDELA, I.; HANEY, C. R.; CHANDA, D.; GEREAU IV, R. W.; ROGERS, J. A. Wireless, battery-free optoelectronic systems as subdermal implants for local tissue oximetry. Science advances, v. 5, n. 3, p. eaaw0873, 2019. Disponível em: https://bit.ly/37uSWnT

ZHANG, Q.; JIANG, D.; XU, C.; GE, Y.; LIU, X.; WEI, Q.; HUANG, L.; REIN, X.; WANG, C.; WANG, Y. Wearable electrochemical biosensor based on molecularly imprinted Ag nanowires for noninvasive monitoring lactate in human sweat. Sensors and Actuators B: Chemical, p. 128325, 2020. Disponível em: https://bit.ly/3g5UM2h

ZHANG, Y.; LIU, C.; LIU, X.; ZHANG, K. A miniaturized circularly polarized implantable RFID antenna for biomedical applications. International Journal of RF and Microwave Computer-Aided Engineering, v. 30, n. 3, p. e22105, 2020. Disponível em: https://bit.ly/3ol68ma 
ZHAO, F., CONZUELO, F., HARTMANN, V., LI, H., STAPF, S., NOWACZYK, M. M., ROGNER, M.; PLIMERÉ, N.; LUBITZ, W.; SCHUHMANN, W. A novel versatile microbiosensor for local hydrogen detection by means of scanning photoelectrochemical microscopy. Biosensors and Bioelectronics, v. 94, p. 433-437, 2017. Disponível em: https://bit.ly/2L6jhkD

ZHAO, W.; TIAN, S.; HUANG, L.; LIU, K.. DONG, L.; GUO, J. A smartphone-based biomedical sensory system. Analyst, v. 145, n. 8, p. 2873-2891, 2020. Disponível em: https://rsc.li/37uTE4x

ZHOU, G.; WANG, Y.; CUI, L. Biomedical sensor, device and measurement systems. Advances in Bioengineering, v. 177, 2015. Disponível em: https://bit.ly/2JNg1d5 
